\title{
The value of cordocentesis in current management of intrauterine patient
}

\author{
Kunochova I, Papcun P, Krizko M Jr, Gabor M, Alfoldi M, Ferianec V \\ 2nd Department of Gynecology and Obstetrics, Faculty of Medicine, Comenius University, \\ Bratislava, Slovakia. ferianec@gmail.com
}

\section{ABSTRACT}

OBJECTIVES: Analyzing the clinical group to evaluate current indications for cordocenteses, their complications and data obtained in further pregnancy management.

METHODS: Retrospective analysis evaluated 92 cordocenteses (diagnostic and therapeutic) performed during the period of 2007-2018. These were performed between 17 and 36 weeks of gestation under ultrasound guidance by a specialist at 2nd Department of Gynecology and Obstetrics, Faculty of Medicine, Comenius University. RESULTS: Out of 92 procedures, 78 were diagnostic and 14 were therapeutic. The diagnostic cordocentesis was successful in $97.4 \%$ and intrauterine therapy was successful in $85.7 \%$. There were $2(2.56 \%)$ diagnostic cordocenteses complicated by fetal demise and $2(14 \%)$ intrauterine demises in therapeutic cordocentesis. The pathological karyotype was detected in $14.5 \%$. Aneuploidia was present in 4 cases (44.4\%), mosaicism in 4 cases $(44.4 \%)$ and triploidia in one case $(11.1 \%)$.

CONCLUSION: Despite of novel molecular genetic technique cordocentesis still plays unreplaceable role in current prenatal diagnosis and treatment. The risk of complications of cordocentesis increases depending on the severity of fetal pathology in pathologic pregnancies. In some situations it can be used as a useful tool for original fetal diagnosis and therapy (Tab. 3, Ref. 20). Text in PDF www.elis.sk.

KEY WORDS: cordocentesis, intrauterine procedure, prenatal diagnosis and treatment, fetus.

\section{Introduction}

Cordocentesis (intrauterine puncture of the umbilical cord that is made to obtain a sample of fetal blood or to supply a drug) is an invasive procedure in fetal medicine, implemented in the second and third trimester of pregnancy. In terms of diagnosis, it is necessary in cases where the fetal genetic, metabolic, hematological, infectological and oxygenation status need to be determined. After receiving an exact diagnosis, it can also serve as a mean of direct fetal treatment - performing fetal transfusions, pharmacological and hormonal treatments. Despite the fact that we currently consider the cordocentesis to be routine, it is necessary to carry it out only in strictly indicated cases and to follow the rate of its early and late complications. Cordocentesis should be performed by an experienced specialist. Strict audits of indications and complications are essential for the use of cordocentesis in prenatal diagnosis and fetal medicine.

We analyzed 92 cordocenteses that were performed at our center. The aim of our study is to provide knowledge on the range of indications and complications of this invasive prenatal diagnostic technique, to evaluate the yield of the data obtained.

2nd Department of Gynecology and Obstetrics, Faculty of Medicine, Comenius University, Bratislava, Slovakia

Address for correspondence: $\mathrm{V}$. Ferianec, MD, PhD, 2nd Department of Gynecology and Obstetrics, Faculty of Medicine, Comenius University, Ruzinovska 6, SK-826 06 Bratislava, Slovakia.

Phone: +421.905385670

\section{Methods}

The study was carried out at the 2nd Department of Gynecology and Obstetrics, Faculty of Medicine, Comenius University. During the period of 2007-2018 a total of 92 cordocentesis procedures were performed, of which 78 were diagnostic and 14 therapeutic. Women at 17 and 36 weeks of gestation who underwent appropriately indicated cordocentesis were consecutively recruited into the study. The procedure was performed after obtaining written consent. Data were retrospectively analyzed. The procedure was performed under an ultrasound - guided freehand technique with preprocedural antibacterial skin preparation, without administration of maternal sedation and prophylactic antibiotics. Local anesthesia was not applied. $22 \mathrm{G}$ needle was used. Depending on the indication for the procedure, gestational age, maternal body habitus, and the distance from skin to target, the length of the needle was also adequately used. Fetal cardiac activity was controlled immediately after the procedure, and further control was performed after 12 to 24 hours. After the procedure there was a reduced physical exercise of at least 12 hours to 24 hours recommended. Bed immobilization was not needed.

\section{Results}

A total of 92 cordocentesis procedures were performed with a success rate of $97.4 \%$ in diagnostic cordocentesis and $85.7 \%$ in intrauterine therapy. The mean gestational age at diagnostic 
Tab. 1. Indication of fetal blood sampling.

\begin{tabular}{lc}
\hline Diagnostic Cordocentesis & $\mathrm{n}(\%)$ \\
\hline Ultrasonography findings & $34(43.5)$ \\
Unclear Result of TAC * & $15(19.2)$ \\
Diagnosis of Anemia & $13(16.6)$ \\
Late Karyotyping ** & $12(15.3)$ \\
Infectious indication & $2(2.5)$ \\
Differential diagnosis of fetal goiter & $1(1.2)$ \\
Determination of intrauterine acidosis parameters of fetus & $1(1.2)$ \\
\hline Total & 78 \\
\hline
\end{tabular}

*TAC - transabdominal amniocentesis - failure of laboratory assessment, unclear result (procedure requiring geneticist), ** late capture according to gestational age

Tab. 2. Intrauterine therapy.

\begin{tabular}{lc}
\hline Therapeutic cordocentesis & $\mathrm{n}(\%)$ \\
\hline Intrauterine transfusion & $12(85.7)$ \\
Intrafetal alcohol chemosclerosis of acardiac twin & $1(7.1)$ \\
Ablation of acardiac twin with Histoacryl & $1(7.1)$ \\
\hline Total & 14 \\
\hline
\end{tabular}

Tab. 3. Number of procedures relative to gestational age.

\begin{tabular}{lcc}
\hline Gestational age & Cordocentesis (n) & Therapy (n) \\
\hline $17-20$ & 8 & 1 \\
$21-24$ & 50 & 5 \\
$25-28$ & 10 & 4 \\
$29-32$ & 7 & 4 \\
$33-36$ & 3 & \\
\hline Total & 78 & 14 \\
\hline
\end{tabular}

cordocentesis was 22 weeks of gestation (range, 17 to 36 weeks). The mean gestational age at intrauterine therapy was 28 weeks of gestation (range, 20 to 31 weeks).The cordocentesis indications and therapeutic indications are summarized in Tables 1 and 2. The number of diagnostic cordocenteses and therapy in the respective gestational age are shown in Table 3.

In this analysis we have specifically evaluated detection of pathological karyotypes (subgroups - unclear TAC result, late karyotyping and suspected ultrasonography morphology). The pathological karyotype was present in $14.5 \%$ by executing a cordocentesis. In the spectrum of genetic diagnoses, aneuploidy was present in four cases $(44.4 \%)$, mosaicism in four cases $(44.4 \%)$ and triploidy in one case $(11.1 \%)$.

Two complications $(2.56 \%)$ were observed during the implementation of diagnostic cordocentesis. Complications led to pregnancy loss. In the first case, the fetus was in the 18th week of gestation with fetal hydrops and in the other case there was fetus with severe intrauterine growth restriction (IUGR) - minus 5/6 weeks of gestation. Two therapeutic complications (14\%) were observed with therapeutic cordocentesis followed by fetal demise. In the first case, this was an extremely early onset of fetal anemia with generalized fetal hydrops at 19th gestational week. Despite the successful application of intrauterine transfusion, the fetus died in utero within 24 hours. In the latter case, intrauterine fetal demise was noticed within 12 hours after alcohol chemosclerosis of an acardiac twin.

\section{Discussion}

In spite of new non-invasive prenatal diagnostic methods such as cell-free DNA determination in non-invasive prenatal diagnosis (NIPT), invasive prenatal diagnosis and especially cordocentesis plays an important role in prenatal diagnosis and fetal medicine. As part of prenatal diagnosis, its role is to ascertain definitive diagnosis that is necessary for further decision-making on pregnancy management. The overall success rate of cordocentesis is reported in $97-98 \%(1,4)$, which is also confirmed by our results $(97.4 \%)$. It is therefore an accepted and relatively safe method of prenatal diagnosis, but its risk of complications is higher compared to other methods. Taking this into account, cordocentesis should be carried out by an experienced specialist who has sufficient experience.

Higher procedure-related complications may occur in high-risk pregnancies, for example in association with intrauterine growth restriction, non-immune fetal hydrops, chromosomal abnormalities. It is reported in $3.2-10.2 \%(1,2,5,6)$. We have to consider some complication as: bleeding from the injection site (20-30 $\%)$, severe fetal bradycardia (5-10\%) in certain cases requiring emergency delivery (4), development of chorioamnionitis, paraumbilical hematoma and vertical transmission of human immunodeficiency virus or hepatitis transmission $(1,3,4)$. Despite the wide spectrum of intrauterine pathology investigated, we achieved only a low percentage of complications reaching the lower limit indicated by world medical sources $(2.56 \%)$.

Less frequently, cordocentesis procedure in terms of the diagnosis, is useful for determination of acidosis parameters in prenatally diagnosed chronic hypoxia of the fetus, predicted on the basis of abnormal umbilical artery Doppler waveforms (7, 8). A definitive diagnosis of fetal thyreopathy in prenatally detected fetal goiter can be defined by determining thyroid hormone serum levels from a fetal blood specimen $(9,10,11,12)$.

The most standard for common procedure within intrauterine therapy in terms of cordocentesis is intrauterine transfusion (IUT). It is the only possibility of fetal treatment in fetal infectious conditions and $\mathrm{Rh}$ iso-immunization (13). The intrauterine hemotherapy of our patients achieved a success rate of $92.3 \%$, compared to data published in the literature which stated up to 86-95\% (13, $14,15)$. Higher IUT complication rates leading to pregnancy loss are often due to high risk pregnancy that requires urgent invasive therapy $(16,17)$. This procedure may be complicated by premature rupture of the membranes and premature labor, with all the risks of prematurity (15). In our group, we observed one case of premature rupture of membranes with subsequent premature labor in pregnancy with generalized fetal hydrops. Other types of intrauterine therapies, such as intraumbilical application of the ablation medium in cardiac twin, remain very rare. In the case of fetal impasse it is the only way to save the fetus $(18,19,20)$.

By analyzing our group we have confirmed the persistence of a wide range of indications for cordocentesis. The analysis also confirmed a low complication rate despite the high severity of intrauterine conditions. Cordocentesis represents an important diagnostic - therapeutic procedure without which prenatal diagnosis and fetal medicine cannot be performed nowadays. 


\section{Learning points}

Cordocentesis is an important invasive prenatal diagnostic technique in fetal medicine.

Cordocentesis is an invasive technique but in skilled hands it is a safe method.

It offers accurate diagnosis which can only be achieved by fetal blood sampling.

Intrauterine therapy performed by cordocentesis can be in lifethreating situations the only one possibility for the fetus.

\section{References}

1. Berry SM et al. Fetal blood sampling. Amer J Obstetr Gynecol 2013; 209 (3): 170-180.

2. Tongsong $\mathbf{T}$ et al. Fetal loss rate associated with cordocentesis at midgestation. Amer J Obstetr Gynecol 2001; 184 (4): 719-723.

3. Johnstone-Ayliffe $\mathbf{C}$ et al. Early procedure-related complications of fetal blood sampling and intrauterine transfusion for fetal anemia. Acta Obstetr Gynecol Scand 2012; 91 (4): 458-462.

4. Guan HB et al. Application of percutaneous cordocentesis in prenatal diagnosis. J China Med Univ 2009; 38 (8): 621-623.

5. Bigelow CA et al. Percutaneous umbilical blood sampling: current trends and outcomes. Eur Obstetr Gynecol Reprod Biol 2016; 200: 98-101.

6. De IJ et al. Predictive factors for fetal tolerance to cordocentesis: A monocentric retrospective study. Gynecol Obstetri Fertil 2012; 40 (12): 734-740.

7. Ferianec $\mathbf{V}$ et al. An alternative management for growth retarded fetus with absent end-diastolic velocity in umbilical artery and normal cardiotocography. Neuroendocrinol Lett 2008; 29 (5): 635.

8. Arduini $\mathbf{D}$ et al. Fetal $\mathrm{pH}$ value determined by cordocentesis: an independent predictor of the development of antepartum fetal heart rate late decelerations in growth retarded fetuses with absent end-diastolic velocity in umbilical artery. J Perinat Med 1996; 24 (6): 601-608.
9. Khamisi S, Lindgren P, Karlsson FA. A rare case of dyshormonogenetic fetal goiter responding to intra-amniotic thyroxine injections. Eur Thyroid J 2014; 3 (1): 51-56.

10. Kim MJ et al. Intra-amniotic thyroxine to treat fetal goiter. Obstetr Gynecol Sci 2016; 59 (1): 66-70.

11. Ferianec $\mathrm{V}$ et al. Prenatal diagnosis and successful intrauterine treatment of severe congenital hypothyroidism associated with fetal goiter. J Obstetr Gynaecol Res 2017; 43 (1): 232-237.

12. Tanase-Nakao K et al. Fetal Goitrous Hypothyroidism and Polyhydramnios in a Patient with Compound Heterozygous DUOXA2 Mutations. Hormone Res Paediatr 2018; 90 (2): 132-137.

13. Prefumo $\mathbf{F}$ et al. Fetal anaemia: diagnosis and management. Best Practice Res Clin Obstetr Gynaecol 2019.

14. Van Kamp IL, Klumper FJCM, Meerman RH, Oepkes D, Scherjon SA, Kanhai HHH. (2004) Treatment of fetal anemia due to redcell alloimmunization with intrauterine transfusions in the Netherlands, 1988-1999. Acta Obstetr Gynecol Scand 2004; 83 (8): 731-737.

15. Van Kamp IL et al. Complications of intrauterine intravascular transfusion for fetal anemia due to maternal red-cell alloimmunization. Amer J Obstetr Gynecol 2005; 192 (1): 171-177.

16. Lindenburg I, Van Kamp IL, Oepkes D. Intrauterine blood transfusion: current indications and associated risks. Fetal Diagn Ther 2014; 36 (4): 263-271.

17. Yinon $\mathbf{Y}$ et al. Early intrauterine transfusion in severe red blood cell alloimmunization. Ultrasound Obstetr Gynecol 2010; 36 (5): 601-606.

18. Ferianec $V$ et al. Successful ablation of acardiac twin with Histoacryl. Taiwanese J Obstetr Gynecol 2016; 55 (1): 109-111.

19. Ferianec $V$ et al. Intrauterinný manažment akardiackých gemín $=$ Intrauterine management of acardiac twins. Akt Gynekol Porod 2014; 6: 10.

20. Corbacioglu A et al. Treatment of twin reversed arterial perfusion sequence with alcohol ablation or bipolar cord coagulation. Internat $\mathrm{J}$ Gynecol Obstet 2012; 117 (3): 257-259.

Received April 8, 2019. Accepted May 14, 2019. 